\title{
Travel Time Prediction under Mixed Traffic Conditions Using RFID and Bluetooth Sensors
}

\author{
Akhilesh Jayan¹, Sasidharan Premakumari Anusha1* \\ 1 Department of Civil Engineering, College of Engineering Trivandrum, Bus Bay, Ambady Nagar, Thiruvananthapuram, \\ 695016 Kerala, India \\ * Corresponding author, e-mail: anushanair@gmail.com
}

Received: 23 January 2019, Accepted: 26 March 2019, Published online: 16 December 2019

\begin{abstract}
Travel time information is an integral part in various ITS applications such as Advanced Traveler Information System, Advanced Traffic Management Systems etc. Travel time data can be collected manually or by using advanced sensors. In this study, suitability of Bluetooth and RFID (Radio Frequency Identifier) sensors for data collection under mixed traffic conditions as prevailing in India is explored. Reliability analysis was carried out using Cumulative Frequency Diagrams (CFDs) and buffer time index along with evaluation of penetration rate and match rate of RFID and Bluetooth sensors. Further, travel time of cars for a subsequent week was predicted using the travel time data obtained from RFID sensors for the present week as input in ARIMA modeling method. For predicting the travel time of different vehicle categories, relationships were framed between travel time of different vehicle categories and travel time of cars determined from RFID sensors. The stream travel time was then determined considering the travel time of all vehicle categories. The R-Square and MAPE values were used as performance measure for checking the accuracy of the developed models and were closer to one and lower respectively, indicating the suitability of the RFID sensors for travel time prediction under mixed traffic conditions. The developed estimation schemes can be used as part of travel time information applications in real time Intelligent Transportation System (ITS) implementations.
\end{abstract}

Keywords

mixed traffic, RFID, bluetooth, ARIMA modeling, travel time prediction, ITS

\section{Introduction}

Travel time is one of the most important types of information required by the commuters during their travel. Travel time can be defined as the average time required by vehicles to transverse a route between any two points of interest. Travel time information is becoming increasingly important for a variety of real-time transportation applications. These real time applications include Intelligent Transport Systems (ITS) systems such as Advanced Traveler Information Systems (ATIS), Advanced Traffic Management Systems (ATMS), Route Guidance Systems (RGS), etc. ITS results in improved safety to drivers, better traffic efficiency, reduced traffic congestion, improved energy efficiency and environmental quality and enhanced economic productivity. ITS systems use various types of sensors to allow gathering of information about the parameters of vehicles and their motion. Now-a-days, sensor networks can be deployed along a road with very little installation and maintenance costs. Most ITS detection technologies were developed for homogeneous traffic flow with lane discipline. Loop detectors were one of the most common detection technology used for data collection all over the world. But the intrusive nature of the loop detector leads to installation problems, maintenance problems, removal of pavement and traffic disruption. Several non-intrusive sensing technologies have been introduced with the development of ITS in the recent past. However, under traffic conditions commonly prevalent in India, where the traffic stream is composed of a number of vehicle categories with varying sizes and speeds operating with minimal lane discipline (referred to as "mixed traffic conditions" in this paper), data collection becomes more challenging and accuracy of many of these sensors become degraded. Few automated traffic data collection and extraction techniques have been well established for mixed traffic conditions.

The development of automated solutions for traffic detection under such traffic conditions is still ongoing. 
Currently, GPS (Global Positioning System) technology is widely used to collect data for the determination of travel time. But in India, only a small number of vehicles are equipped with GPS devices. Data collection using Bluetooth sensor is more efficient and less expensive way in the determination of travel time. There have been a number of studies internationally that shows that Bluetooth data is a reliable source of travel time information. However, anecdotal evidence suggests that most commuters will turn their Bluetooth devices after use in order to prevent unauthorized access or to extend battery life in India, which largely affect the sample. Hence, it would be interesting to explore Bluetooth as a source of travel time data in India. Measurement of travel time using RFID (Radio Frequency Identifier) sensors is a novel technology in the field of travel time studies. RFID sensors detects the vehicles which have RFID tags affixed in it. In India, RFID tags have been installed in cars manufactured after the year 2014. Moreover, all classes of vehicles have RFID tags optionally installed for Electronic Toll Collection (ETC) on the National Highway network in India.

Only limited studies have explored the reliability of travel time estimated using Bluetooth and RFID sensors under mixed traffic conditions in India. Mathew et al. (2016) conducted a study on variation in travel time along two different routes and developed various reliability indices such as mean, standard deviation and buffer time index to quantify the reliability. The ability of Bluetooth sensors for capturing the travel time and monitoring the traffic conditions along the study stretch was carried out in this study. Gudhishala et al. (2016) conducted a feasibility study on using Bluetooth Probe Detection System (BPDS) to determine travel time of urban road network. This study focused on the possibility of using BPDS for measuring overall congestion, trends in congestion, identify location of hotspots and measurement of level of congestion. The findings of study were promising indicating that BPDS can be effectively used for the determination of travel time and measure the congestion in terms of indices such as travel time index, planning time index and travel delay. Haghani et al. (2010) introduced Bluetooth sensors for data collection of freeway ground truth travel time. The authors explained the concept of vehicle identification and travel time estimation using Bluetooth signatures. Issues related to error analysis, filtering of raw matched data were discussed. Results showed that the Bluetooth technology is a promising method for collecting high-quality travel time data.
Araghi et al. (2014) evaluated the effect of the size of detection zone and short range antennas for detection of Bluetooth enabled devices. It was found that, on average, Bluetooth-enabled devices were detected $80 \%$ of the time while passing a sensor location. The impact of location ambiguity caused by the size of the detection zone was evaluated using geo-coded Bluetooth data. Results showed that more than $80 \%$ of the detection events were recorded within the range of $100 \mathrm{~m}$ from the sensor center line. It was also shown that short-range antennas detect Bluetooth-enabled devices in a location closer to the sensor, thus providing a more accurate travel time estimate. However, the smaller the size of the detection zone, the lower is the penetration rate, which could itself influence the accuracy of estimates. The authors suggested that there is a trade-off between acceptable level of location ambiguity and penetration rate for configuration and coverage of the antennas while considering the detection of Bluetooth enabled devices.

The data collected using sensors can be used for the prediction of travel time. Kumar et al. (2011) devised a model based approach using the Kalman filtering technique to predict stream travel time from GPS enabled public transit. Only two wheeled vehicles were considered in this study as they constituted a major proportion in the study area. The prediction scheme was corroborated using field data collected by carrying GPS units in two-wheelers traveling along with the buses under consideration. The travel time estimates from the model were compared with the manually observed travel times and the results were promising. Mandal et al. (2011) devised methods to analyze the traffic movement and congestion pattern with the help of active RFID technology. Kumar and Vanajakshi (2015) developed a short term prediction model for traffic flow using SARIMA model. Three days data were collected consecutively for the development of models. Study stretch selected was, a three lane road in Chennai, India. Differentiation was done on the input data to make it stationary and suitable SARIMA models were identified by plotting Auto Correlation Function (ACF) and Partial Auto Correlation Function (PACF) graphs. R-software was used for the prediction and model parameters were found using maximum likelihood method. The predicted values obtained using the models were compared with the actual flow values using MAPE (Mean Absolute Percentage Error) and the developed model indicated high accuracy. Bhaskar et al. (2013) conducted a study on forecasting of short term future travel time. The data 
used for the prediction had seasonality and SARIMA models were used for the prediction. Travel time was determined from data collected using Bluetooth sensors. Road network in Brisbane City was selected for the study. Data were collected for eight months and based on the characteristics of data, SARIMA modelling was selected for prediction. Accuracy of the model, its robustness and transferability were evaluated by comparing the real and predicted values on three sites within Brisbane network.

From the literature review, it can be observed that effectiveness of Bluetooth and RFID sensors for travel time prediction under mixed traffic conditions have rarely been attempted. Hence this study aims at exploring the possibility of Bluetooth and RFID sensors as data collection technique and using them for the development of travel time prediction models for ITS applications. The methodology of study, study site and data collection, data analysis, and the development of prediction models are presented in the sections below.

\section{Methodology of the study}

This paper focuses on the application of Bluetooth sensors and RFID sensors to capture the MAC (Media Access Control) Ids and Unique Ids of vehicles to determine the travel time along a selected road stretch in Trivandrum, Kerala. Penetration rate and match rates of Bluetooth and RFID sensors along the selected stretches were determined. Using the data obtained from sensors, reliability in travel time were analyzed by drawing CFD (Cumulative Frequency Diagram) and Buffer Time index for each time interval of day in the study stretch. These reliability measures were used to analyze the variation in travel time along the selected stretch. Travel time of cars for subsequent week was predicted using travel time data obtained from RFID sensors for the present week as input in ARIMA modeling. Relationships were framed between travel time of cars and travel time of all category of vehicles to determine the stream travel. The flow chart illustrating the methodology of the study is shown in Fig. 1.

\section{Study site and data collection}

The study stretch (Fig. 2) selected was an undivided four lane section in an urban arterial spanning between two locations Karyavattom and Pongumoodu in Trivandrum, Kerala. The total route length of the study stretch is $5.4 \mathrm{~km}$. The entry point was selected at Karyavattom and exit point was selected at Pongumoodu.
Selection of study stretch for data collection

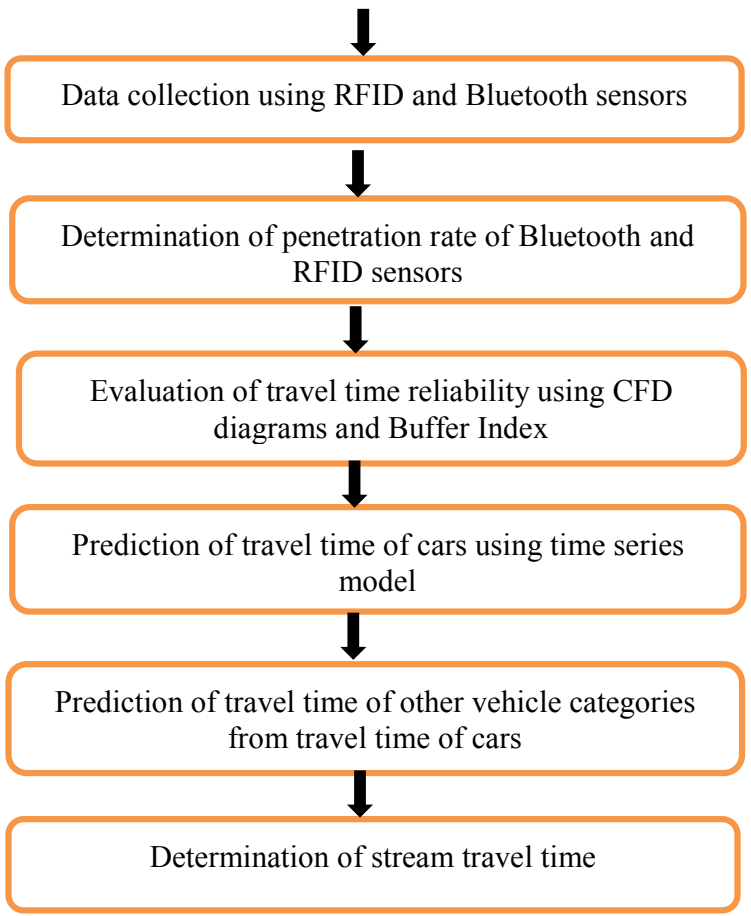

Fig. 1 Study methodology.

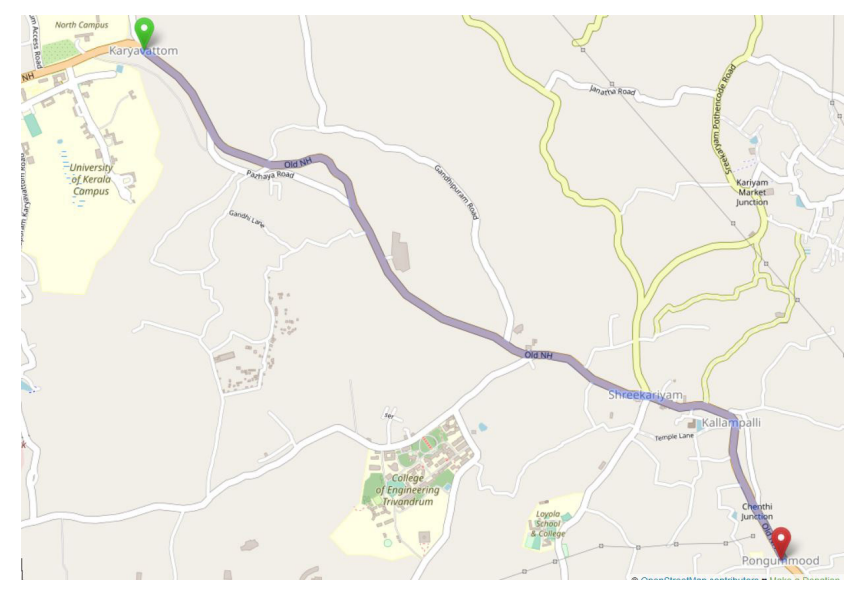

Fig. 2 Route map of study stretch (Source: Open Street Maps).

Data were collected from 9:00 am to 6:00 pm for two weeks from 11/02/2018 to 24/02/2018. Data collection units comprising of Bluetooth Sensor, RFID reader, Raspberry $\mathrm{Pi}$ and GPRS connectivity were deployed at both locations. The Bluetooth sensors records the MAC ID of the Bluetooth devices within the vehicles and the RFID sensors records the unique ID of RFID tags of the cars in the vehicle stream along with the time at which these ID's were detected. Videographic survey was carried out, on $19^{\text {th }}$ February 
and $26^{\text {th }}$ February from $11: 00$ to $13: 00$ and $16: 00$ to $18: 00$, to record the traffic volume at the entry and exit of the study stretch for determination of penetration rate and match rate of the sensors. Fig. 3 shows the photograph of data collection unit deployed at one of the study location.

The raw data recorded by RFID and Bluetooth sensors are sent to the Traffic Intelligence Server (TIS) (Proprietary software by ITSPE) hosted on the cloud. Fig. 4 shows the screenshot of TIS. The recorded data are send to the database through 4G connectivity. The data can be accessed by logging on to the TIS by using unique user ID and password.

\section{Data analysis}

The recorded data were downloaded from the TIS in Microsoft excel format. Vehicles with same unique ID at the entry and exit location were matched and sorted. Fig. 5 shows the sample data that were sorted by matching the vehicle IDs detected at the entry and exit locations. This also includes the time stamp of the vehicles indicating the detection time of vehicles at the entry and exit locations. The travel time was calculated using Eq. (1):

$$
T T=t_{e}-t_{s},
$$

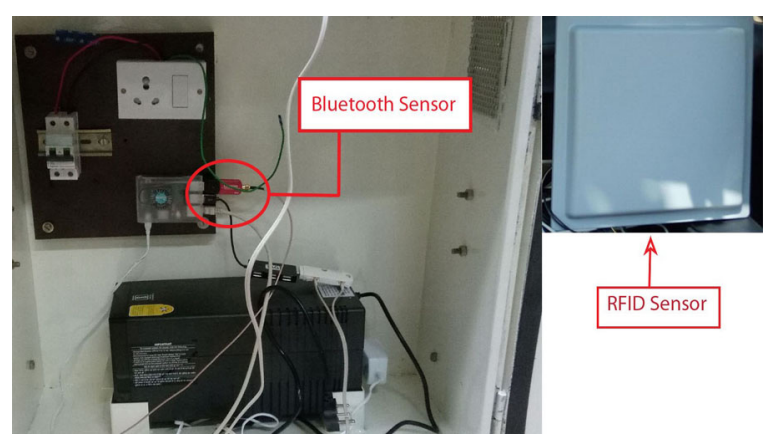

Fig. 3 Data collection unit.

\section{Traffic Intelligence Server}

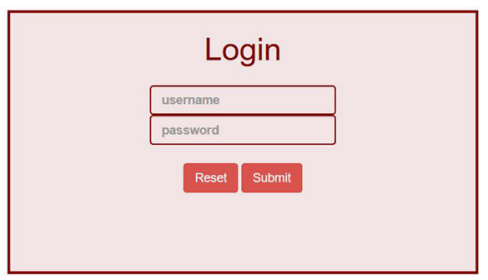

Fig. 4 Screenshot of Traffic Intelligence Server. where $T T$ is the travel time, $t_{e}$ is the time stamp at the exit and $t_{s}$ is the time stamp at the entry.

Fig. 6 shows the travel time variation of data collected on $14^{\text {th }}$ February. It can be seen that some data points obtained in the matching stage are unacceptable for several reasons. These data points are unacceptable due to observations with lesser travel time or observations which are far away from the average values of previous travel time data. These data points are considered as outliers. The outliers in the travel time data need to be removed for further analysis. There are several methods for the detection of outliers depending upon the type of data. In this study, MAD (Median Absolute Deviation) technique was used for the removal of outliers. There are other outlier removal methods such as overtaking rule method (Robinson and Polak, 2006) which uses the temporal structure of the data to filter out erroneous travel time records. However, since the data obtained from the study stretch does not include any excessive delays due to intersections or accidents, MAD technique was adopted.

MAD was determined for the three time periods such as morning peak (9:00 to 12:00), off-peak (12:00 to 15:00) and evening peak (15:00 to 18:00). The median of the travel time for the different time periods was found. The difference between each of the travel time values in this period and the median was calculated. These differences were expressed as their absolute values, and a new median is calculated to yield the MAD. MAD was calculated using Eq. (2). The MAD was then multiplied by an empirically derived constant ( 3 in this study) to obtain the weighted MAD value. The median of travel times of these time periods were then added and subtracted from the weighted MAD value to obtain the upper and lower threshold range of travel times. If a travel time value is out of this range, that value is classified as an outlier. This method is more effective than other techniques like mean and standard deviation for the removal of outliers (Araghi et al., 2014).

$\operatorname{MAD}(T T)=$ Median $t t(i)-\operatorname{Median}(T T)$,

where MAD (TT) is the mean absolute deviation of travel time for the selected time period, $t t(i)$ is the travel time of vehicle, $i$ in this time period and Median (TT) is the median of travel time of all vehicles within the selected time period.

The MAD method fixes threshold values for maximum and minimum values of travel time, and travel time values which are not within the threshold values were removed. Travel time data after removal outliers is shown in Fig. 7. It can be observed that the resulting travel times after the 


\begin{tabular}{|c|c|c|c|c|c|c|c|c|}
\hline No: & Start Time & Unique Id & Device & No: & End Time & Unique Id & Device & Time(min) \\
\hline DET001 & 9:07:30 AM & 35303338.00 & RFID & DET002 & 9:27:38 AM & 35303338.00 & RFID & 20.1 \\
\hline DET001 & 9:08:49 AM & 171901432630.00 & RFID & DET002 & $9: 20: 20 \mathrm{AM}$ & 171901432630.00 & RFID & 11.5 \\
\hline DET001 & 9:20:41 AM & 691300311630.00 & RFID & DET002 & 9:30:15 AM & 691300311630.00 & RFID & 9.6 \\
\hline DET001 & 9:21:50 AM & 350В01812800 & RFID & DET002 & 9:30:18 AM & 350В01812800 & RFID & 8.5 \\
\hline BL001 & 9:23:30 AM & 00:EC:0A:9A:35:FE & BLUETOOTH & BL002 & 9:35:40 AM & 00:EC:0A:9A:35:FE & BLUETOOTH & 12.2 \\
\hline DET001 & $9: 30: 15 \mathrm{AM}$ & 71701050990.00 & RFID & DET002 & $9: 40: 46 \mathrm{AM}$ & 71701050990.00 & RFID & 10.5 \\
\hline DET001 & 9:32:04 AM & 130601240610.00 & RFID & DET002 & 9:41:13 AM & 130601240610.00 & RFID & 9.1 \\
\hline DET001 & 9:36:25 AM & 410B01762770 & RFID & DET002 & 9:46:14 AM & 410B01762770 & RFID & 9.8 \\
\hline DET001 & 9:38:02 AM & 421102701130.00 & RFID & DET002 & 9:48:19 AM & 421102701130.00 & RFID & 10.3 \\
\hline DET001 & 9:41:00 AM & 20328A520318 & RFID & DET002 & 9:51:19 AM & 20328A520318 & RFID & 10.3 \\
\hline DET001 & 9:46:52 AM & 41102052620.00 & RFID & DET002 & 9:56:22 AM & 41102052620.00 & RFID & 9.5 \\
\hline BL001 & 9:46:53 AM & $08: 73: B 2: 97: 62: 61$ & BLUETOOTH & BL002 & 9:56:47 AM & 08:73:B2:97:62:61 & BLUETOOTH & 9.9 \\
\hline DET001 & 9:49:14 AM & 20328A520316 & RFID & DET002 & 9:56:56 AM & 20328A520316 & RFID & 7.7 \\
\hline DET001 & 9:49:20 AM & 910402562180.00 & RFID & DET002 & 9:56:09 AM & 910402562180.00 & RFID & 6.8 \\
\hline DET001 & 9:51:23 AM & 140201992640.00 & RFID & DET002 & 10:00:19 AM & 140201992640.00 & RFID & 8.9 \\
\hline DET001 & 9:53:11 AM & 50302210460.00 & RFID & DET002 & 10:10:10 AM & 50301462150.00 & RFID & 17.0 \\
\hline DET001 & 9:57:38 AM & 991601110240.00 & RFID & DET002 & 10:11:05 AM & 991601110240.00 & RFID & 13.5 \\
\hline DET001 & 9:59:19 AM & 710601562280.00 & RFID & DET002 & $10: 10: 56 \mathrm{AM}$ & 710601562280.00 & RFID & 11.6 \\
\hline DET001 & 9:59:26 AM & 140902110660.00 & RFID & DET002 & 10:09:57 AM & 140902110660.00 & RFID & 10.5 \\
\hline BL001 & 9:59:30 AM & BC:02:4A:3C:5C:90 & BLUETOOTH & BL002 & 10:08:16 AM & $\mathrm{BC}: 02: 4 \mathrm{~A}: 3 \mathrm{C}: 5 \mathrm{C}: 90$ & BLUETOOTH & 8.8 \\
\hline DET001 & 9:59:32 AM & 71200261070.00 & RFID & DET002 & 10:07:26 AM & 71200261070.00 & RFID & 7.9 \\
\hline DET001 & 10:01:45 AM & 1002371080.00 & RFID & DET002 & 10:10:01 AM & 1002371080.00 & RFID & 8.3 \\
\hline DET001 & 10:02:42 AM & 20328A52031A & RFID & DET002 & 10:13:02 AM & 20328A520312 & RFID & 10.3 \\
\hline DET001 & 10:05:02 AM & 140601931800.00 & RFID & DET002 & 10:14:08 AM & 140601931800.00 & RFID & 9.1 \\
\hline DET001 & $10: 06: 08 \mathrm{AM}$ & 71002250950.00 & RFID & DET002 & $10: 15: 14 \mathrm{AM}$ & 71002250950.00 & RFID & 9.1 \\
\hline
\end{tabular}

Fig. 5 Screenshot of sample data of travel time.

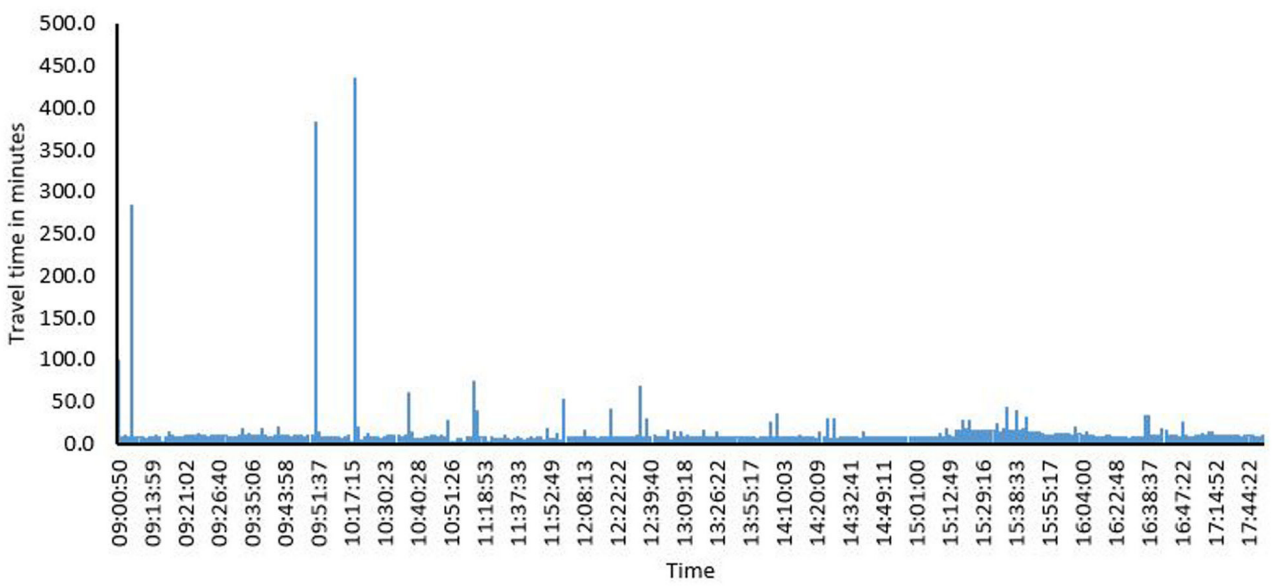

Fig. 6 Travel time data of $14^{\text {th }}$ February.

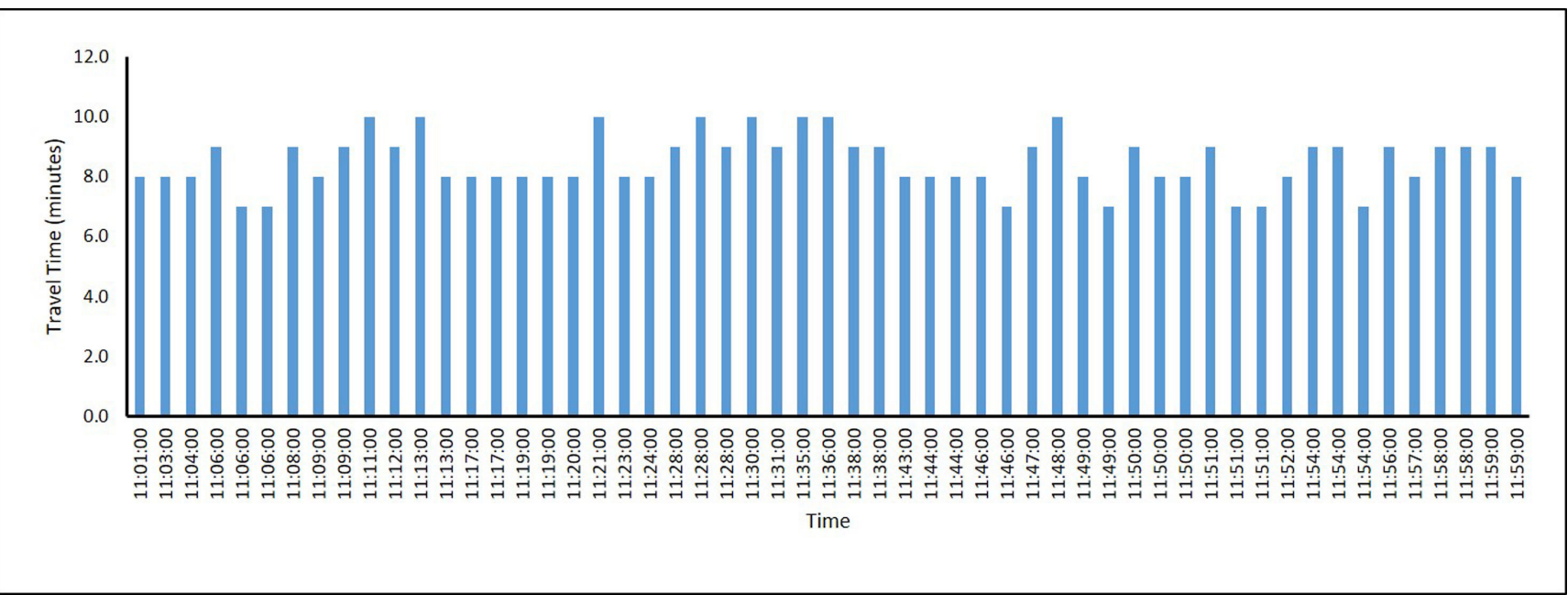

Fig. 7 Travel time after removal of outliers. 
removal of outliers falls within the reasonable range and were used for further analysis. Fig. 8 represents the travel time data from $11^{\text {th }}$ February to $17^{\text {th }}$ February after the removal of outliers.

\subsection{Penetration rate and match rate}

A penetration rate analysis was carried out to analyze the percentage of vehicles identified by each sensor. Video cameras were located at both the locations to record the traffic volume on $19^{\text {th }}$ February. The volume count at each locations was obtained by manual data extraction. The penetration rate was determined using Eq. (3).

$P R=\frac{N_{s}}{N_{t}}$,

where $P R$ is the penetration rate $N_{s}$ is the total number of sensors detected and $N_{t}$ is the total number of vehicles that passed the location. Table 1 shows the penetration rate for RFID and Bluetooth sensors at each location.

From Table 1, it can be observed that the penetration rate of RFID is higher compared to the Bluetooth sensors. Lower penetration rate of Bluetooth sensors may be due to the change in visibility settings in the commuters Bluetooth device.

The match rate was determined using Eq. (4) to check the percentage of vehicles being matched at the two locations along the selected road stretch.

$$
M_{R}=\frac{N_{m}}{N_{d}}
$$

where $M_{R}$ is the match rate, $N_{m}$ is the number of vehicles matched and $N_{d}$ is the number of vehicles detected using the sensors.
Table 1 Penetration rate of sensors at each of the study locations

\begin{tabular}{lccc}
\hline \multirow{2}{*}{ Location } & Time & \multicolumn{2}{c}{ Penetration Rate (\%) } \\
& & RFID & Bluetooth \\
\hline \multirow{2}{*}{ Karyavattom } & $11: 00$ to $13: 00$ & 14.0 & 1.8 \\
& $16: 00$ to $18: 00$ & 11.1 & 1.4 \\
Pongumood & $11: 00$ to $13: 00$ & 12.5 & 1.6 \\
& $16: 00$ to $18: 00$ & 10.3 & 1.5 \\
\hline
\end{tabular}

The match rate of RFID sensors was found to be $50.2 \%$ and $1.2 \%$ for Bluetooth sensors, indicating that RFID sensors have better match rate and can be suitable for data collection for travel time studies under mixed traffic conditions.

\subsection{Reliability studies}

Travel time reliability studies are useful in evaluating the quality of traffic service. In this study Cumulative Frequency Diagrams (CFDs) were drawn and buffer time index was determined using the data obtained from RFID and Bluetooth sensors to evaluate and quantify the reliability of travel time in the selected route.

\subsubsection{Reliability studies using CFD}

The Cumulative Frequency Diagrams (CFD's) are plots with the cumulative frequency of travel times on the $\mathrm{x}$-axis and their corresponding percentiles on the y-axis. The CFD's were plotted for data collected from 11/02/2018 to 17/02/2018. Time sessions were divided into 9:00 to 12:00, 12:00 to 15:00 and 15:00 to 18:00. A total of three sets of CFD's were plotted for a day with time periods ranging from 09:00-12:00 12:00-15:00 and 15:00-18:00, in order to capture the variations during peak and off peak conditions.

Fig. 9 shows CFD diagrams of week end and CFD diagrams of week days are shown in Fig. 10. From the CFD

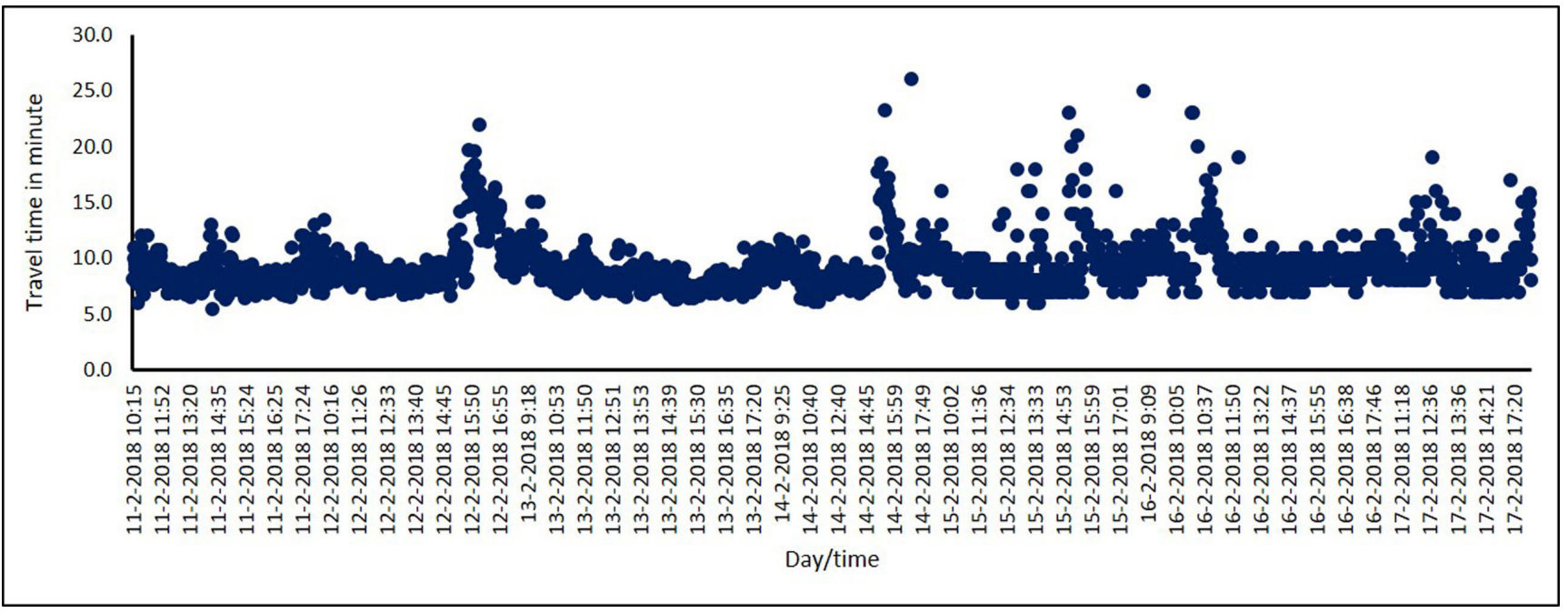

Fig. 8 Travel time data of a week after removal of outliers. 
9:00 to $12: 00$
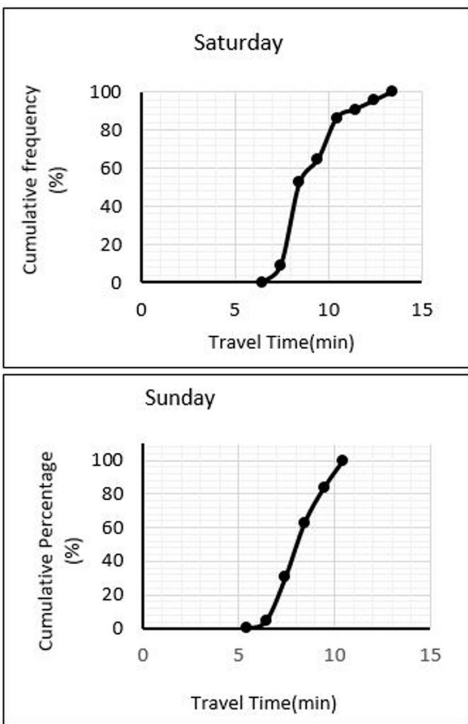

$12: 00$ to $15: 00$
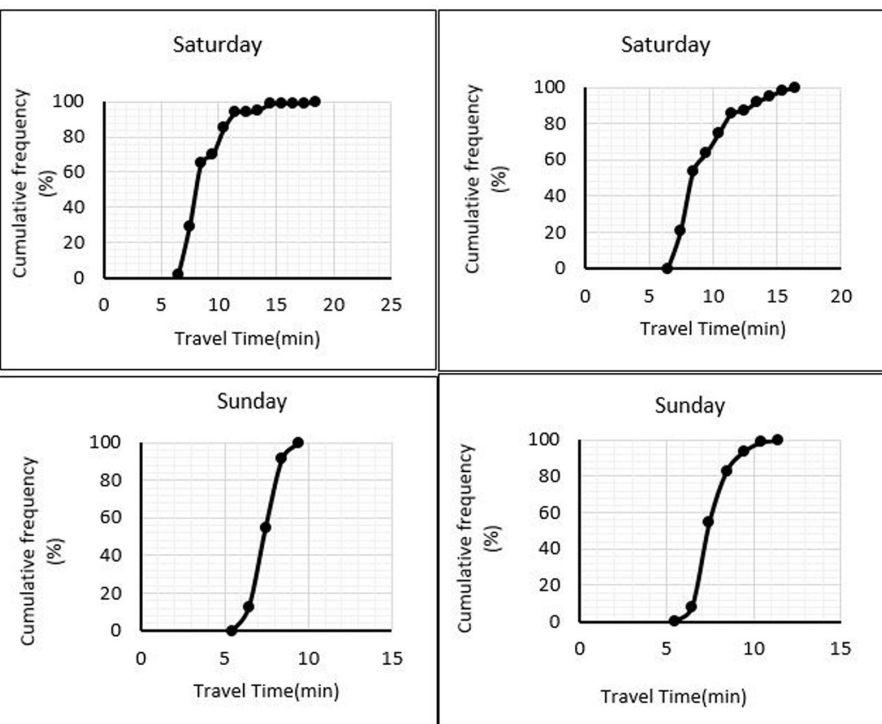

Fig. 9 Daily assessment of travel time reliability on weekend.

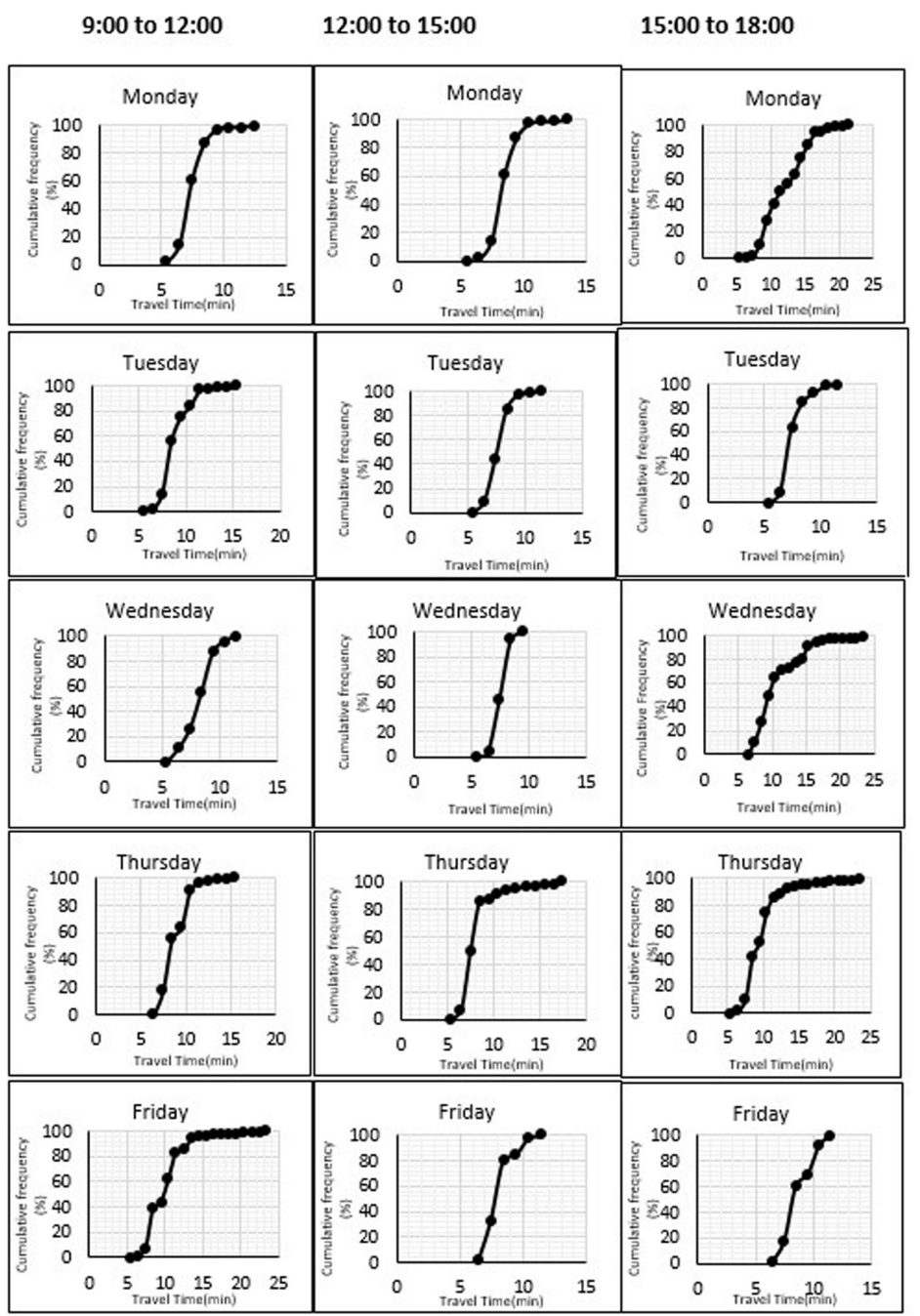

Fig. 10 Daily assessment of travel time reliability on working days 
diagrams of working day it can be observed that the travel time of working day is more between 15:00 to 18:00 and the travel time is less during 12:00 to 15:00. From the graphs, it can be observed that the peak hours of weekend is between 09:00 to $12: 00$ and 15:00 to 18:00 and off peak hours are between 12:00 to 15:00. By analyzing the weekend data, it can be observed that the travel time on Saturday is less compared to other working day and it is even more less on Sunday. On Sunday the off peak hour is between 09:00 to 15:00 and peak hour is between 15:00 to 18:00. Thus, it can be seen that the travel time varies for each day of same week along the same route.

\subsubsection{Reliability studies using buffer time index}

Reliability in travel time reflects the performance of the road network and is also used to measure the effects of congestion in road network. There are several measures for quantifying travel time reliability. In this study, buffer time index is used as performance measure to evaluate the travel time reliability.

Buffer Time: It indicates the extra time the traveller should add to the average travel time to ensure on time arrival at the destination (1).

Buffer Time Index

$$
=\frac{\left(95^{\text {th }} \text { percentile time }- \text { Mean travel time }\right)}{\text { Mean travel time }} \text {, }
$$

where $95^{\text {th }}$ percentile time is the travel time below which $95 \%$ of vehicle travel. Table 2 shows the buffer time index for morning peak (09:00 to 12:00), off peak (12:00 to 15:00) and evening peak (15:00 to 18:00) of working days and buffer time index for weekend data for morning peak, off peak and evening peak for week end data are shown in Table 3. It can be seen that the buffer time index increases with increase in $95^{\text {th }}$ percentile time. The travel time of the particular time interval in a selected route can be obtained by multiplying the buffer time index with the average travel time.

\subsection{Travel time prediction model}

From the data analysis, it can be seen that Bluetooth sensors detects only lesser percentage of the total vehicles passing through the study stretch. Hence, the travel time data obtained using the RFID sensors were only used for the model development. Since the RFID captures only travel time of cars, models were first developed for prediction of travel time of cars. Time series model was used for prediction since travel time data resembles the time series data. ARIMA (Autoregressive Integrated Moving Average) model was used for the prediction of travel time.
Table 2 Buffer time index for working day data

\begin{tabular}{|c|c|c|c|}
\hline Date & Time & $\begin{array}{l}95^{\text {th }} \text { Percentile Time } \\
\text { (Minute) }\end{array}$ & $\begin{array}{l}\text { Buffer } \\
\text { Index }\end{array}$ \\
\hline \multirow{3}{*}{ 12-Monday } & $09: 00$ to $12: 00$ & 9.2 & 0.03 \\
\hline & $12: 00$ to $15: 00$ & 10.2 & 0.26 \\
\hline & $15: 00$ to $18: 00$ & 16.3 & 0.30 \\
\hline \multirow{3}{*}{ 13-Tuesday } & $09: 00$ to $12: 00$ & 11.2 & 0.20 \\
\hline & $12: 00$ to $15: 00$ & 9.1 & 0.12 \\
\hline & $15: 00$ to $18: 00$ & 9.2 & 0.14 \\
\hline \multirow{3}{*}{ 14-Wednesday } & $09: 00$ to $12: 00$ & 10.3 & 0.17 \\
\hline & $12: 00$ to $15: 00$ & 8.5 & 0.05 \\
\hline & $15: 00$ to $18: 00$ & 16.3 & 0.46 \\
\hline \multirow{3}{*}{ 15-Thursday } & $09: 00$ to $12: 00$ & 10.9 & 0.18 \\
\hline & $12: 00$ to $15: 00$ & 12.1 & 0.44 \\
\hline & $15: 00$ to $18: 00$ & 14.8 & 0.44 \\
\hline \multirow{3}{*}{ 16-Friday } & $09: 00$ to $12: 00$ & 13.9 & 0.31 \\
\hline & $12: 00$ to $15: 00$ & 10.2 & 0.20 \\
\hline & $15: 00$ to $18: 00$ & 10.6 & 0.16 \\
\hline \multicolumn{4}{|c|}{ Table 3 Buffer time index for weekend data } \\
\hline Date & Time & $\begin{array}{l}95^{\text {th }} \text { Percentile } \\
\text { (Minute) }\end{array}$ & Buffer Index \\
\hline \multirow{3}{*}{ 11-Sunday } & 09:00 to $12: 00$ & 10.2 & 0.19 \\
\hline & $12: 00$ to $15: 00$ & 8.6 & 0.09 \\
\hline & $15: 00$ to $18: 00$ & 9.5 & 0.17 \\
\hline \multirow{3}{*}{ 17-Saturday } & 09:00 to $12: 00$ & 12.5 & 0.30 \\
\hline & $12: 00$ to $15: 00$ & 11.8 & 0.28 \\
\hline & $15: 00$ to $18: 00$ & 14.3 & 0.46 \\
\hline
\end{tabular}

The general model is written as $\operatorname{ARIMA}(p, d, q)$ where $p$ is the order of auto regression, $d$ is the degree of differencing, $q$ is the order of moving average involved.

In order to apply ARIMA the input series needs to be stationary, indicating that it should have a constant mean, variance, and autocorrelation through time. Therefore, the series first needs to be differenced until it is stationary. The number of times the series needs to be differenced to achieve stationarity is reflected in the $d$ parameter. In order to determine the necessary level of differencing, the plot of the data and autocorrelation should be examined. Significant changes in level (strong upward or downward changes) usually require first order non seasonal $($ lag $=1)$ differencing and strong changes of slope usually require second order non seasonal differencing. By examining the Auto Correlation Function (ACF) and Partial Auto Correlation Function (PACF) plot of the travel time data used in this study, it was observed that there were no strong upward or downward changes indicating that differencing is not necessary for the data. A plot of $\mathrm{ACF}$ and PACF for a sample data is shown in Fig. 11. 


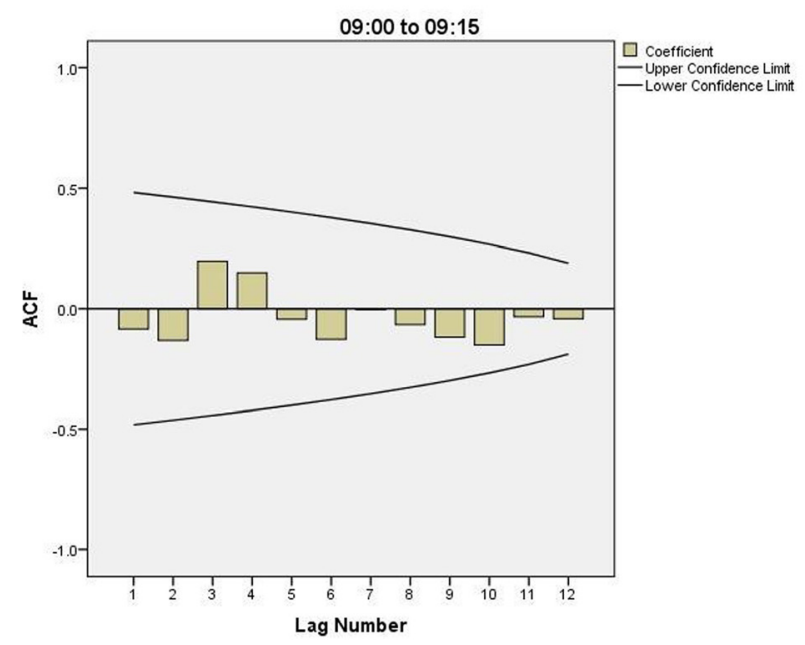

(a)

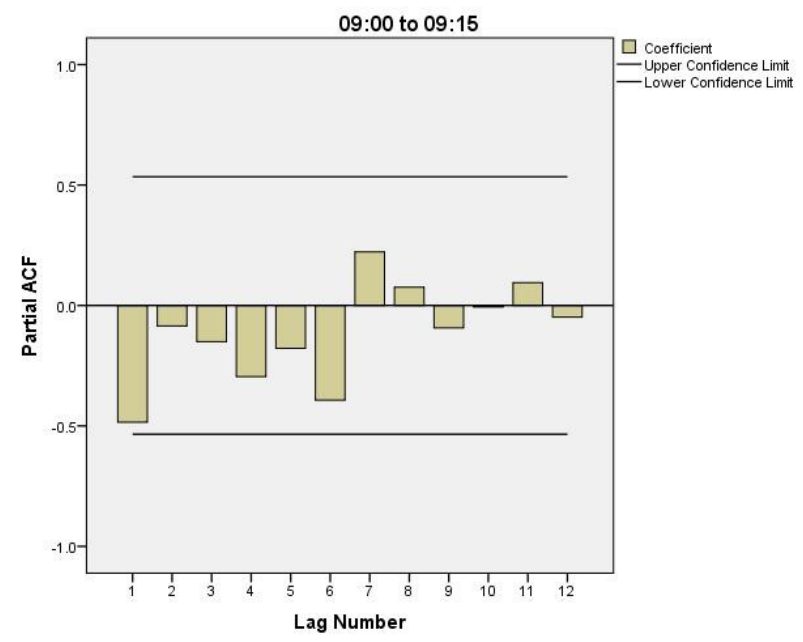

(b)

Fig. $11 \mathrm{ACF}$ and PACF plots.

The plots of ACF and PACF helps in identifying the type of model. If the ACF dies down and PACF cut off after a few lag, then AR (Auto Regressive) model will be selected. While if ACF cuts off after a few lags and PACF dies down, then MA (Moving Average) model is selected. On the other hand, if both ACF and PACF dies down then, ARIMA is adopted.

For the prediction of travel time, the time stamps were first divided into 15-min interval. Auto Correlation Function (ACF) and Partial Auto Correlation Function (PACF) graphs were plotted using Statistical Package for the Social Sciences (SPSS) software for each time interval. Fig. 11 shows the ACF and PACF graph of travel time between 09:00 to 09:15.

From Fig. 11, it can be seen that there is a gradual decay in the ACF graph after lag 7 and gradual decay in the PACF graph after lag 2 indicating AR and MA model. Since the data is found to be stationary, no differencing is done. Hence, the model can be written as ARIMA (7, 0, 2). Likewise, ACF and PACF graphs for all time intervals were plotted and the corresponding models were selected for the prediction. By analyzing the ACF and PACF graphs for each time interval, ARIMA models were developed for the prediction of travel time for the consecutive week. Travel time data from $11^{\text {th }}$ February to $17^{\text {th }}$ February was used as input for prediction of travel time using ARIMA model. The travel time data from $18^{\text {th }}$ to $24^{\text {th }}$ February taken from 09:00 to 18:00 hours including both peak as well as off peak hour data were used for validation of the developed models.

The predicted travel times were compared with the actual travel times for all days of the subsequent week from $18^{\text {th }}$ February to $24^{\text {th }}$ February. Figs. 12 and 13 show the comparison of predicted and actual values of travel time data for two sample days, Tuesday and Saturday respectively. It can be observed that the predicted travel time matches reasonably well with the actual travel time. The accuracy of the model was checked using MAPE

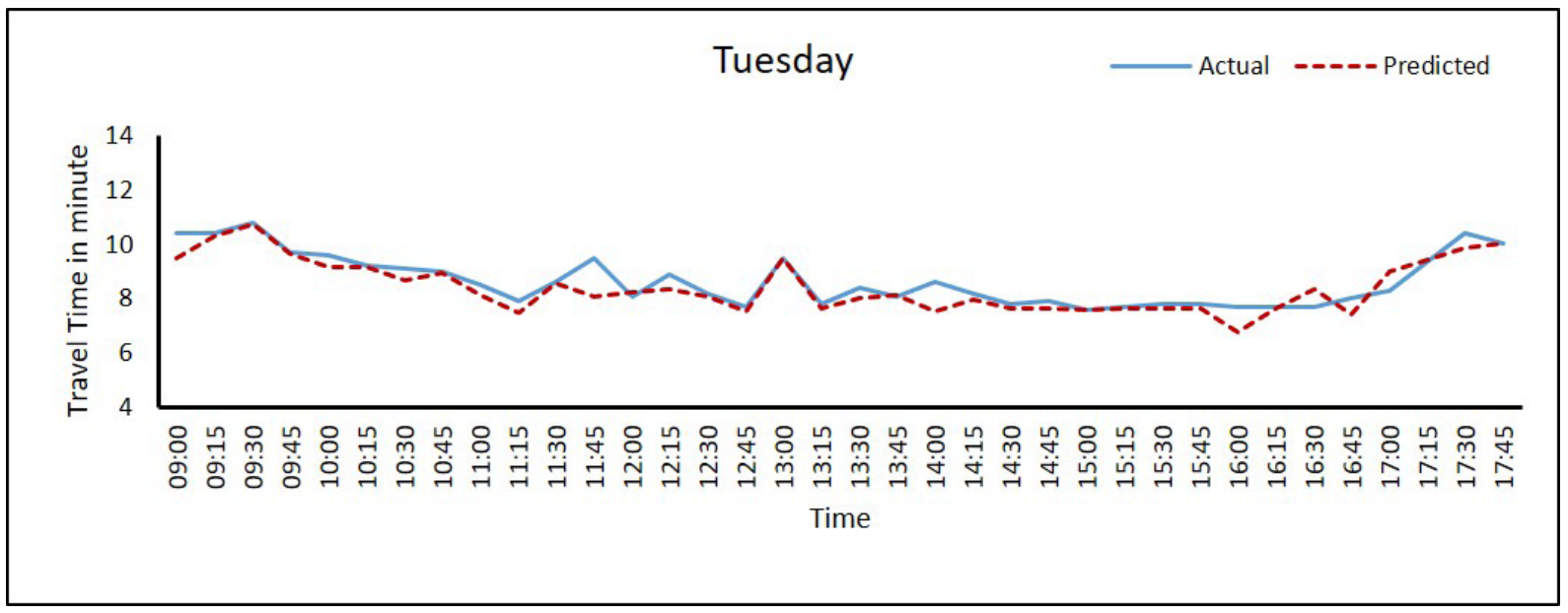

Fig. 12 Predicted and observed travel time data for Tuesday. 


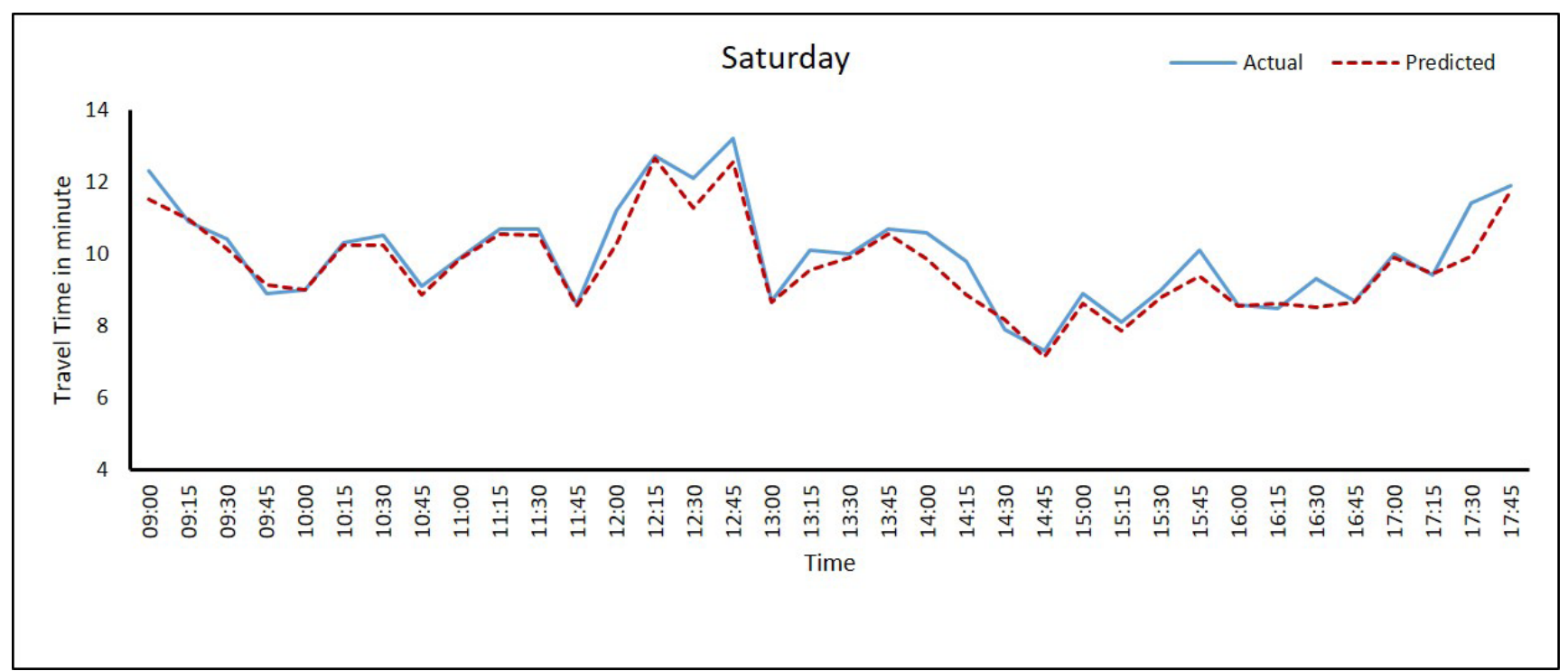

Fig. 13 Predicted and observed travel time data for Saturday

(Mean Absolute Percentage Error) and R-Square values. MAPE is obtained as

$$
\text { MAPE }=\left(\frac{1}{N} \sum_{k=1}^{N} \frac{\left|T T_{p r e d}(k)-T T_{a c t}(k)\right|}{T T_{a c t}(k)}\right) \times 100,
$$

where $T T_{\text {pred }}(k)$ and $T T_{a c t}(k)$ are the predicted and the measured travel time of the vehicles in the study stretch during the $k^{\text {th }}$ interval of time with $N$ being the total number of time intervals. According to Lewis' scale of judgement of forecasting accuracy (Klimberg et al., 2010), any forecast with a MAPE value of less than $10 \%$ is considered highly accurate, $11 \%$ - $20 \%$ is good, $21 \%$ - $50 \%$ is reasonable and $51 \%$ or more is inaccurate. R-Square values closer to one indicate high level of accuracy of developed models.

Table 4 shows the MAPE and R-Square values of the predicted result for each day of week. It can be observed that the MAPE values are less than 10 and R-Square values closer to one, indicating that the model has a very good accuracy and can be used for travel time prediction of cars.

Table 4 MAPE and R-Square values of the predicted travel time

\begin{tabular}{lcc}
\hline Date & R-Square & MAPE $(\%)$ \\
\hline Sunday & 0.89 & 3.06 \\
Monday & 0.74 & 7.92 \\
Tuesday & 0.84 & 3.52 \\
Wednesday & 0.77 & 7.31 \\
Thursday & 0.92 & 3.01 \\
Friday & 0.81 & 3.12 \\
Saturday & 0.75 & 6.32 \\
\hline
\end{tabular}

\subsubsection{Prediction of stream travel time}

Travel time of the traffic stream depends upon the travel time of different categories of vehicles such as two-wheelers, three-wheeler, cars, Light Motor Vehicles (LMVs) and buses moving in the stream. Travel time of each vehicle category was determined by drawing relationship between travel time of car determined using RFID sensors and travel time of other categories of vehicles determined from videographic survey. Relationships were drawn for peak and off peak hours due to high variation in travel time. The manual data extraction of travel time is time consuming and tedious and hence travel time of different vehicle categories were predicted for Monday of the subsequent week using the travel time of Monday of the present week as input. Travel time data collected on $19^{\text {th }}$ February was used for developing the regression models and data collected on $26^{\text {th }}$ February was used for validation. Figs. 14 and 15 show the relationships obtained between travel time of cars and other categories of vehicles for off peak (11:00 to $13: 00)$ and peak (16:00 to $18: 00)$ hours.

Regression equations to estimate the travel times of each of the vehicle categories for peak and off-peak hours are shown in the Table 5.

The travel time of different categories of vehicles were predicted for $26^{\text {th }}$ February by using the travel time of car predicted through ARIMA modeling in the developed regression equations. The predicted travel time was compared with the actual travel times that were manually extracted from video. Figs. 16-19 show the comparison 


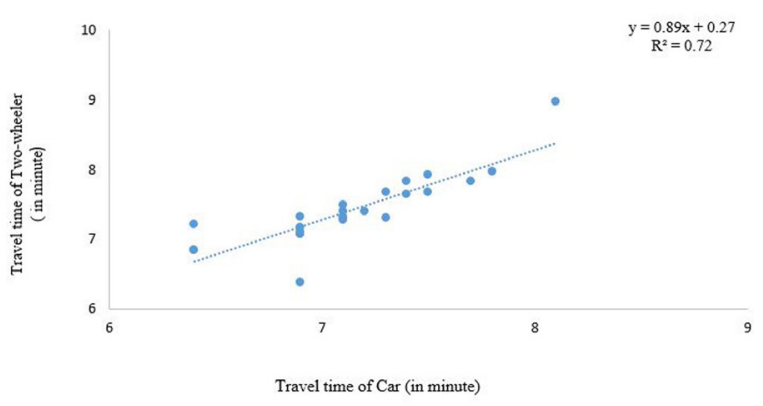

(a)

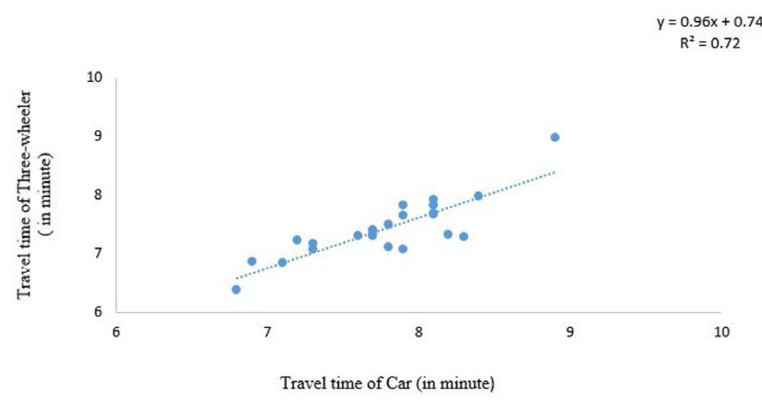

(b)

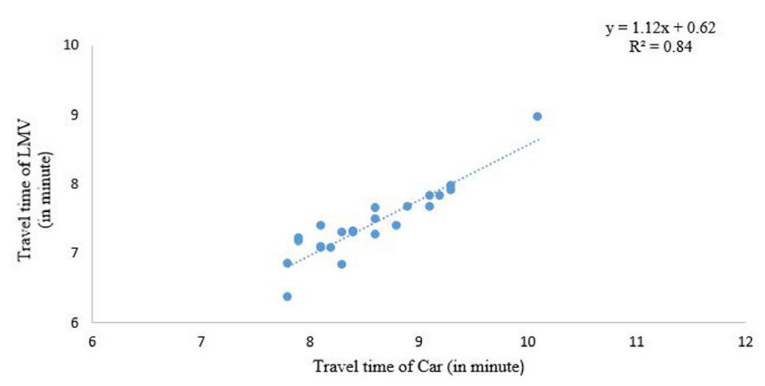

(c)

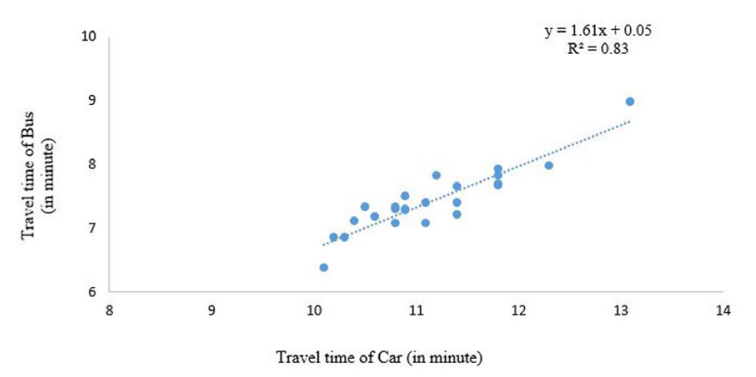

(d)

Fig. 14 Relationships obtained between cars and other vehicle categories (11:00 to 13:00)

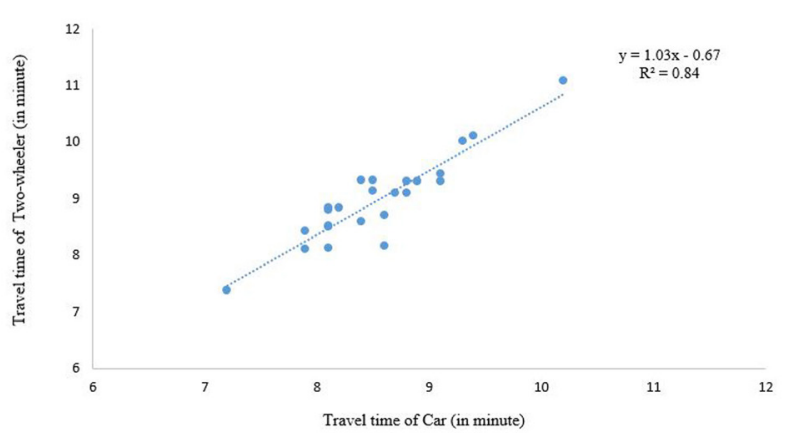

(a)

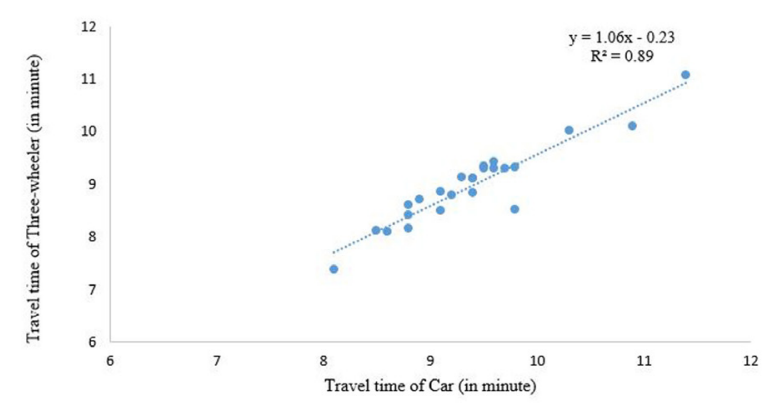

(b)

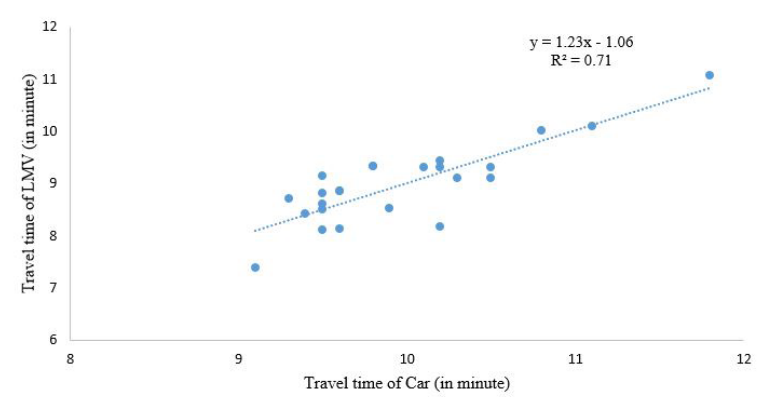

(c)

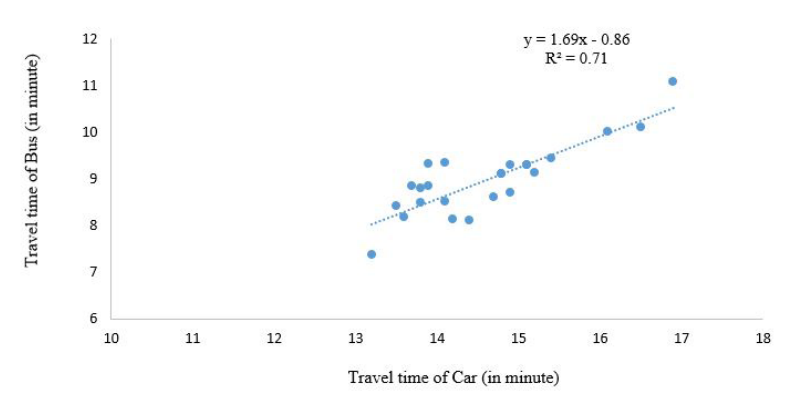

(d)

Fig. 15 Relations obtained between cars and other vehicle categories (16:00 to $18: 00)$. 
Table 5 Regression equations for determining travel time of different vehicle categories

\begin{tabular}{lcc}
\hline Vehicle category & Time interval & Equation \\
\hline \multirow{2}{*}{ Two wheeler } & $11: 00$ to $13: 00$ & $T T_{2 w}{ }^{*}=0.89 T T_{\mathrm{Car}}{ }^{*}+0.27$ \\
& $16: 00$ to $18: 00$ & $T T_{2 w}=1.03 T T_{\mathrm{Car}}-0.67$ \\
\hline \multirow{2}{*}{ Three wheeler } & $11: 00$ to $13: 00$ & $T T_{3 w}{ }^{*}=0.96 T T_{\mathrm{Car}}+0.74$ \\
& $16: 00$ to $18: 00$ & $T T_{3 w}=1.06 T T_{\mathrm{Car}}-0.23$ \\
\hline \multirow{2}{*}{ LMV } & $11: 00$ to $13: 00$ & $T T_{\mathrm{LMV}}{ }^{*}=1.12 T T_{\mathrm{Car}}+0.62$ \\
\hline \multirow{2}{*}{ Bus } & $16: 00$ to $18: 00$ & $T T_{\mathrm{LMV}}=1.23 T T_{C a r}-1.06$ \\
\hline
\end{tabular}

* $T T_{2 w}$ - Travel time of Two-wheeler, $T T_{C a r}$ - Travel time of car,

$T T_{3 w}$ - Travel time of Three-wheeler, $T T_{\mathrm{LMV}}$ - Travel time of LMV, $T T_{B u s}^{3 w}$ - Travel time of Bus.

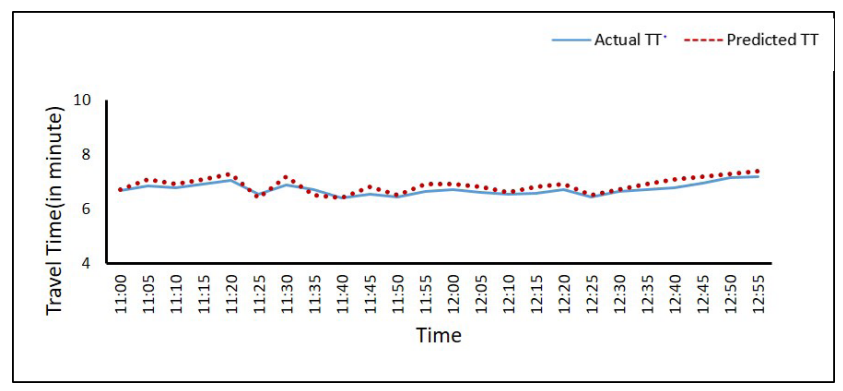

Fig. 16 Comparison of predicted travel time of two-wheeler (off peak).

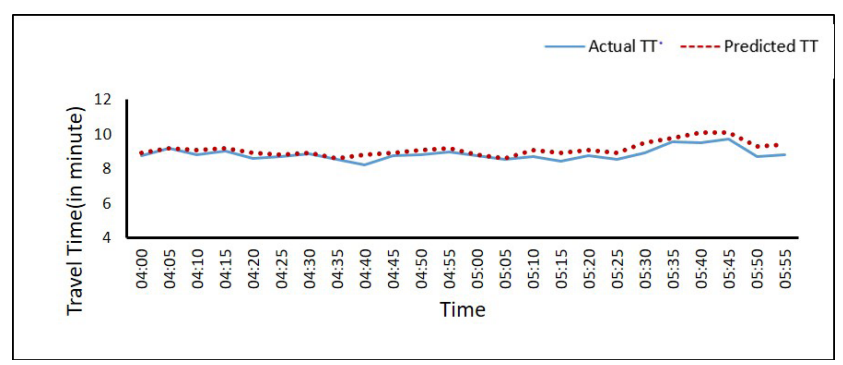

Fig. 17 Comparison of predicted travel time of two-wheeler (peak).

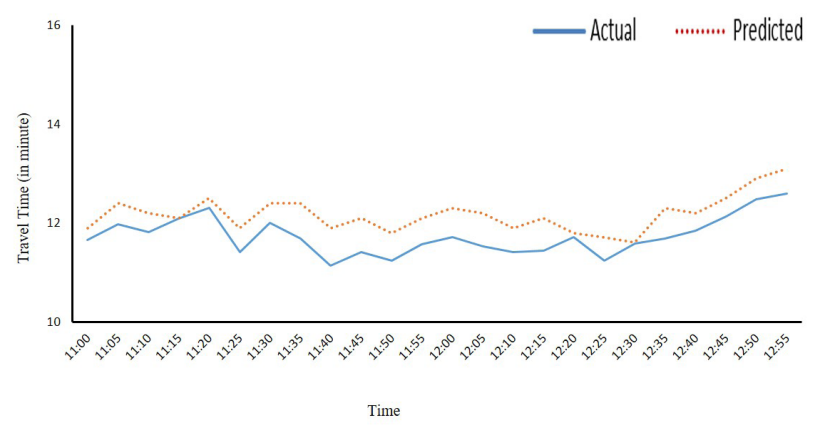

Fig. 18 Comparison of predicted travel time of bus (off peak).

of the predicted and actual travel time for two wheeler and bus during peak and off-peak hours. Table 6 shows the R-Square and MAPE values obtained for all the vehicle categories. The lower values of MAPE indicates good estimation of travel time.

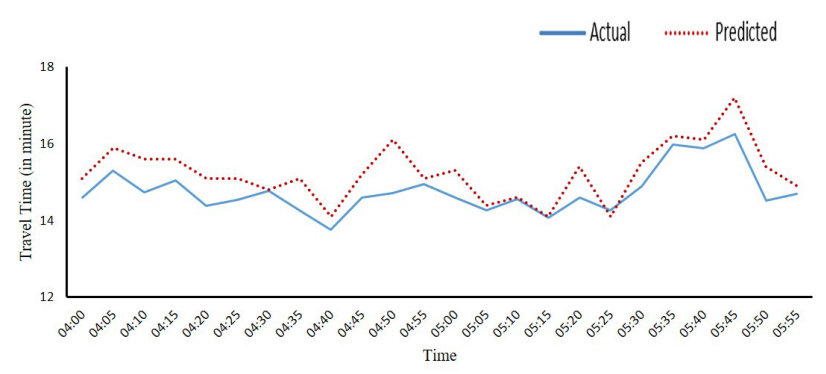

Fig. 19 Comparison of predicted travel time of bus (peak).

Table 6 R-Square values and MAPE of travel time prediction models for different vehicle categories

\begin{tabular}{llcc}
\hline Vehicle category & Time interval & R-Square & MAPE (\%) \\
\hline \multirow{2}{*}{ Two wheeler } & $11: 00$ to $13: 00$ & 0.751 & 6.31 \\
& $16: 00$ to $18: 00$ & 0.863 & 3.14 \\
\hline \multirow{2}{*}{ Three wheeler } & $11: 00$ to $13: 00$ & 0.871 & 3.12 \\
& $16: 00$ to $18: 00$ & 0.833 & 3.85 \\
\hline \multirow{2}{*}{ Car } & $11: 00$ to $13: 00$ & 0.809 & 3.12 \\
& $16: 00$ to $18: 00$ & 0.841 & 3.37 \\
\multirow{2}{*}{ LMV } & $11: 00$ to $13: 00$ & 0.892 & 3.09 \\
& $16: 00$ to $18: 00$ & 0.680 & 10.12 \\
\hline \multirow{2}{*}{ HMV } & $11: 00$ to $13: 00$ & 0.839 & 3.62 \\
& $16: 00$ to $18: 00$ & 0.852 & 3.19 \\
\hline
\end{tabular}

Further, the stream travel time was determined by taking the average of travel times taken by all vehicle categories in the stream. Stream travel time was determined for two sets of time interval. Figs. 20 and 21 show comparison of stream travel time with actual travel time determined from video camera by manual data extraction. Table 7 represents the MAPE and R-Square values obtained for the stream travel time. It can be observed that MAPE values were lower and R-Square values were closer to one, indicating that the predicted stream travel time is accurate.

\section{Summary and conclusions}

Real time information of travel time will help the commuters to plan their trip effectively and overcome the traffic delays. If travel time can be predicted, it can be used in various ITS applications like ATIS and ATMS. Many ITS applications that can provide real time information of travel time to the public can be developed using automated data obtained from sensors. The accuracy of the information provided through such applications depends on the accuracy of the prediction scheme, which in turn depends on the data obtained from these sensors. The prediction of travel time under mixed traffic conditions is a challenging task due to various vehicle categories and lane-less traffic movement. The suitability of RFID and Bluetooth sensors 


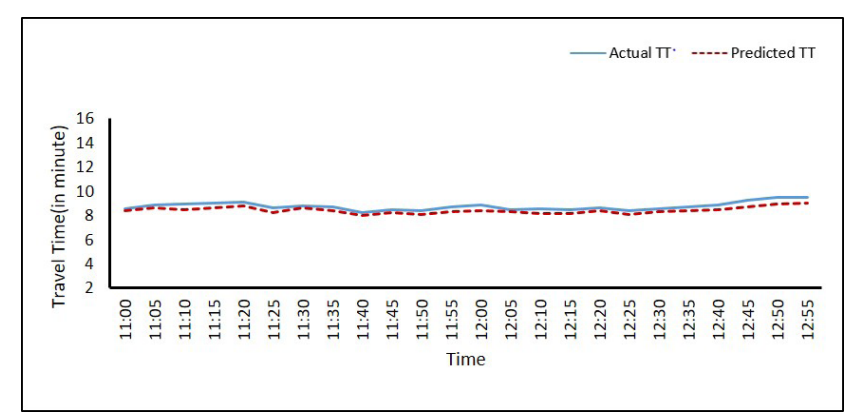

Fig. 20 Comparison of stream travel time (off peak hour).

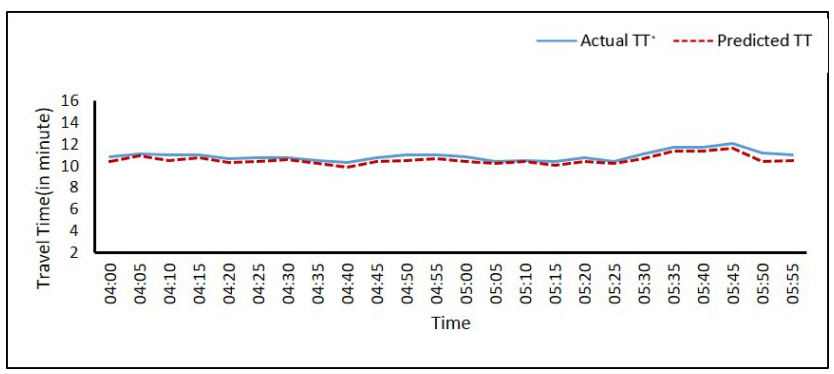

Fig. 21 Comparison of stream travel time (peak hour).

Table 7 MAPE and R-Square values of the stream travel time

\begin{tabular}{lcc}
\hline Time & R-Square & MAPE $(\%)$ \\
\hline Off-peak hour & 0.835 & 3.79 \\
Peak hour & 0.871 & 3.12 \\
\hline
\end{tabular}

\section{References}

Araghi, B. N., Olesen, J. H., Krishnan, R., Christensen, L. T., Lahrman, H. (2014) "Reliability of Bluetooth Technology for Travel Time Estimation", Journal of Intelligent Transportation Systems, 19(3), pp. $240-255$. https://doi.org/10.1080/15472450.2013.856727

Bhaskar, A., Chung, E., Khoei, A. M. (2013) "Travel Time Prediction on Signalised Urban Arterials by Applying SARIMA Modelling on Bluetooth Data", In: Australasian Transport Research Forum, Brisbane, Australia, pp. 1-18. [online] Available at: https://eprints. qut.edu.au/63176/ [Accessed: 26 March 2019]

Gudishala, R.,Wilmot, C., Mokkapatti, A. (2016) "Travel Time Estimation Using Bluetooth", National Center for Intermodal Transportation for Economic Competitiveness, Mississippi. [online] Available at: https://trid.trb.org/view/1400765 [Accessed: 26 March 2019]

Haghani, A., Hamedi, M., Sadabadi, K. F., Young, S., Tarnoff, P. (2010) "Data Collection of Freeway Travel Time Ground Truth with Bluetooth Sensors", Transportation Research Record, Washington, D.C., 2160(1), pp. 60-68.

https://doi.org/10.3141/2160-07

ITS Planners and Engineers, ITSPE "Traffic Intelligence Server", Hyderabad, India. [online] Available at: http:/www.itspe.co.in/ [Accessed: 26 March 2019] for travel time prediction under mixed traffic conditions was explored in this study. Based on the data analysis, it can be concluded that RFID sensors can be used as a data collection technique for travel time studies in mixed traffic conditions due to the higher penetration and match rate, compared to Bluetooth sensors. Development of prediction models for various vehicle categories and stream travel time with good accuracy indicates that the developed schemes can be used as part of the travel time information applications in real time ITS implementations.

\section{Scope for future work}

This study had not considered intersection delay, accident delay, delay due to weather condition etc. due to the unavailability of more number of sensor units. These factors can also be considered for the prediction of travel time for a traffic network.

\section{Acknowledgements}

The authors acknowledge the support for this study by Transportation Research Centre (TRC) set up at College of Engineering, Trivandrum, Kerala through State Budget allotment 2016-17 by the Kerala Government vide letter no. L4/30202/16/DTE dated 23/09/2016. The authors also acknowledge Dr. Rajesh Krishnan, CEO of ITS Planners and Engineers for the technical support provided for conducting the study.

Klimberg, R. K., Sillup, G. P., Boyle, K. J., Tavva, V. (2010) "Forecasting performance measures - what are their practical meaning?", In: Lawrence, K., Klimberg, R. (eds.) Advances in Business and Management Forecasting, Vol. 7, Emerald Group Publishing Limited, Bingley, UK, pp. 137-147. https://doi.org/10.1108/S1477-4070(2010)0000007012

Kumar, V. S., Vanajakshi, L., Subramanian, S. (2011) "A Model Based Approach to Predict Stream Travel Time Using Public Transit as Probes", In: IEEE Intelligent Vehicles Symposium (IV), BadenBaden, Germany, pp. 101-106. https://doi.org/10.1109/IVS.2011.5940413

Kumar, V. S., Vanajakshi, L. (2015) "Short-term traffic flow prediction using seasonal ARIMA model with limited input data", European Transport Research Review, 7, pp. 1-9. https://doi.org/10.1007/s12544-015-0170-8

Mathew, J., Vanajakshi, L., Bullock, D., Sharma, A. (2016) "Investigation of the Use of Bluetooth Sensors for Travel Time Studies under Indian Traffic Conditions", Transportation Research Procedia, 17, pp. 213-222.

https://doi.org/10.1016/j.trpro.2016.11.077 
Mandal, K., Sen, A., Chakraborty, A., Roy, S., Batabyal, S., Bandyopadhyay, S. (2011) "Road traffic congestion monitoring and measurement using active RFID and GSM technology", In: $14^{\text {th }}$ International IEEE conference on Intelligent Transportation Systems, Washington, D.C., USA, pp. 1375-1379.

https://doi.org/10.1109/ITSC.2011.6082954
Robinson, S., Polak, J. (2006) "Overtaking Rule Method for the Cleaning of Matched License-Plate Data", ASCE Journal of Transportation Engineering, 132(8), pp. 609-617.

https://doi.org/10.1061/(ASCE)0733-947X(2006)132:8(609) 